\title{
Asociación entre consumo de vodka y tasa de mortalidad relacionada con el alcohol, a nivel agregado, utilizando series temporales
}

\author{
YurI E. Razvodovsky \\ Grodno State Medical University, Department of Psychiatry. Bielorrusia. \\ Enviar correspondencia a: \\ Yury E. Razvodovsky. Tel: (0152) 33-53-41, e-mail: razvodovsky@grsmi.univel.by
}

\section{RESUMEN}

Antecedentes: La asociación entre el nivel de consumo de alcohol per cápita y el índice de mortalidad relacionada con el consumo de alcohol está muy bien documentada. Tenemos muchos menos datos, sin embargo, sobre el efecto de una bebida específica de alcohol en el índice de mortalidad. Objetivo: Calcular el efecto de una bebida específica de alcohol en el índice de mortalidad relacionada con el consumo de alcohol. Medidas: En Bielorrusia, las tendencias en los diferentes tipos de índice de mortalidad relacionada con el consumo de alcohol (a causa de cirrosis hepática, intoxicación alcohólica, alcoholismo y psicosis alcohólicas) entre 1970 y 1999 fueron analizadas en relación con las tendencias en el nivel de consumo per cápita de diferentes tipos de bebidas alcohólicas, mediante el análisis de series temporales. Resultados: Dicho análisis demostró el efecto directa y estadísticamente significativo de los cambios en el consumo de vodka per cápita sobre los índices de mortalidad relacionada con el alcohol. Al mismo tiempo, apenas se observó la menor correlación entre el nivel total de consumo de alcohol y los diferentes tipos de índices de mortalidad vinculada al alcohol. Conclusión: Los resultados del presente estudio refuerzan la idea de que el índice de mortalidad vinculada al consumo de alcohol tiende a ser más sensible a los cambios experimentados en el consumo de vodka per cápita que al nivel total de consumo de alcohol. La principal evidencia que nos lleva a esta conclusión es la asociación probada y significativa entre los índices de mortalidad vinculados al consumo de alcohol y el nivel de consumo de vodka per cápita. El resultado de este estudio indica, asimismo, que la mortalidad por intoxicación alcohólica es un indicador preferente de los daños vinculados al alcohol en los países donde predomina el modelo de consumo que persigue la embriaguez.

Palabras clave: consumo de alcohol, mortalidad relacionada con el consumo de alcohol, análisis de series temporales.

\section{ABSTRACT}

Background: The association between alcohol consumption level per capita and alcohol-related mortality rate is well documented. Considerably less is known concerning beveragespecific effect of alcohol on mortality rate. Aim: To estimate the beverage-specific effect of alcohol on alcohol-related mortality rate. Measurements: Trends in different types of alcohol-related mortality rate (due to liver cirrhosis, poisoning by alcohol, alcoholism and alcoholic psychosis) from 1970-1999 in Belarus were analyzed in relation to trends in the level of different types of alcoholic beverages consumption per capita applying time series analysis. Results: The analysis demonstrated a positive and statistically significant effect of changes in vodka per capita consumption on alcohol-related mortality rates. At the same time, hardly any correlation is observed between the total level of alcohol consumption and different types of alcohol-related mortality rates. Conclusion: The results of present study give support to the idea that alcohol-related mortality rate tends to be more responsive to changes in vodka consumption per capita than in total level of alcohol consumption. The main evidence for this conclusion is that a positive and significant association between alcohol-related mortality rates and level of vodka consumption per capita was revealed. The outcome of this study also suggests that alcohol poisoning mortality is a preferable marker of alcohol-related harm in the countries with prevailing intoxication-oriented drinking pattern.

Key words: alcohol consumption, alcohol-related mortality, time-series analysis.

\section{INTRODUCCIÓN}

I consumo abusivo de alcohol está asociado al deterioro de la salud física y psíquica del ser humano (Viallant, 1983). El alcohol puede causar problemas agudos cuando se consume ocasionalmente en grandes cantidades, como es el caso de una intoxicación alcohólica. Las consecuencias crónicas son el resultado de un largo período de consumo abusivo de alcohol. Aquí encontraríamos, en primer 
lugar, la adicción al alcohol y las psicosis alcohólicas, que se consideran consecuencias psico-biológicas directas del consumo abusivo de alcohol. Por otra parte, también estarían los trastornos somáticos crónicos, en cuya etiología el alcohol juega un importante papel, como es el caso de la cirrosis hepática (Edwards, 1994; Skog, 1988). Sabemos que el nivel de mortalidad debido a estas razones es un indicador estándar de la prevalencia de los problemas relacionados con el consumo de alcohol en la sociedad. Con la ayuda de este indicador, podemos identificar las diferencias que existen entre unos países y otros en el nivel de los problemas relacionados con el consumo de alcohol. Es evidente que la mortalidad causada por el alcoholismo y las psicosis alcohólicas están en este grupo. La asociación entre el consumo abusivo de alcohol y la cirrosis hepática es, asimismo, indudable (Lelbach, 1976; Smart\&Mann, 1992). El riesgo de mortalidad resulta ser una función más o menos exponencial del consumo de alcohol. En los últimos años, esta relación ha sido estudiada a fondo y descrita en numerosas publicaciones científicas. Así, por ejemplo, la clara y evidente asociación entre el nivel de consumo de alcohol per cápita y el nivel de cirrosis hepática fue probada por algunos análisis de series temporales realizados en distintos países (Thorsen, 1990). Estudios posteriores indicaron también la existencia de un estrecho vínculo entre la cirrosis y el nivel de consumo. De ese modo, los análisis de series temporales basados en datos estadísticos y recogidos en catorce países europeos demostraron el efecto real y estadísticamente significativo de los cambios en el consumo de alcohol per cápita sobre los cambios en la mortalidad por cirrosis en trece países para los hombres y en nueve países para las mujeres (Ramstedt, 2001). Estos resultados sugieren que un cambio en el nivel total de consumo de alcohol afecta la mortalidad por cirrosis en diferentes culturas del alcohol. En algunas investigaciones se analizó el efecto retardado en el tiempo (Skog, 1984; Norstrpom, 1987). Eso significa que la variación en el índice de mortalidad puede disminuir tras la variación del nivel de consumo de alcohol. Esta no-correspondencia puede deberse al hecho de que un gran numero de enfermedades se desarroIlan únicamente después de abusar del alcohol durante un largo período de tiempo. Un período latente tiene un efecto retardado en el tiempo. Así pues, según bastantes estudios, el nivel total de consumo de alcohol parece ir directamente asociado con el índice de mortalidad relacionada con el alcohol. Tenemos muchos menos datos sobre el efecto de una bebida alcohólica específica en el índice de mortalidad por cirrosis hepática. Es difícil generalizar sobre el efecto de una bebida alcohólica específica sobre el índice de mortalidad por cirrosis hepática a nivel de población. Cinco países (Australia, Canadá, Nueva Zelanda, Reino Unido y Estados Unidos) realizaron análisis de series temporales a nivel agregado anual sobre el consumo total de alcohol, cerveza, vino y licores y sobre todas las causas del índice de mortalidad por cirrosis entre 1953 y 1993. Los resultados de esta investigación indicaron que el consumo de licores, más que de vino o cerveza, está asociado a la mortalidad por cirrosis en este grupo de países (Kerr y col., 2000). El papel de los modelos de consumo de alcohol en la asociación alcohol-mortalidad es un tema muy discutido. La evidencia del cruce cultural sugiere que en los países "no prohibicionistas", donde el vino domina la estructura de consumo de alcohol, el nivel de mortalidad debido a problemas crónicos relacionados con el alcohol, como por ejemplo las cirrosis hepáticas, es más elevado. Sin embargo, en los países "prohibicionistas", donde la cultura del alcohol se decanta por los licores, predomina la mortalidad debida a problemas agudos, como es el caso de una intoxicación alcohólica (Edwards y col., 1994). La evidencia descriptiva indica también una marcada pendiente norte-sur en el índice de mortalidad por cirrosis hepática, siendo más elevada en el sur que en el norte. En cuanto a la intoxicación alcohólica, el modelo geográfico resultó ser opuesto al de la cirrosis, pues el índice más elevado de mortalidad por esa causa se encontró en el norte, y el menos elevado en el sur (Ramstedt, 2001). Varios estudios han concluido que el elevado índice de mortalidad por intoxicación alcohólica en Finlandia y Polonia puede atribuirse al modelo de consumo de alcohol que persigue la embriaguez (Poicolainen, 1977, Makela y col., 1981). Así, pues, los problemas agudos relacionados con el consumo de alcohol están asociados en mucha mayor medida con el modelo de consumo de alcohol que con el nivel total de consumo. Al mismo tiempo, el índice de problemas crónicos relacionados con el consumo de alcohol viene determinado por el nivel total de consumo de alcohol.

Este artículo se centra en los análisis de series temporales de la dinámica del nivel de consumo de vodka per cápita, y de los diferentes tipos de índices de mortalidad relacionadas con el consumo de alcohol (mortalidad por alcoholismo o psicosis alcohólicas, mortalidad por intoxicación alcohólica, mortalidad por cirrosis hepática) en Bielorrusia, entre 1970 y 1999.

\section{MATERIALESY MÉTODOS}

Todos los datos que figuran en el artículo proceden de los informes anuales del Ministerio de Estadística de Bielorrusia, entre 1970 y 1999. Los niveles de mortalidad se indican en un ratio de 1 por cada 100.000 habitantes. El consumo de alcohol per cápita (en litros de alcohol puro) se ha calculado en base al consumo total de alcohol determinado por las estadísticas de venta, dividido por la población total. En el período de estudio se han empleado dos revisiones ICD diferentes: ICD-8 e ICD-9. Los códigos ICD para las causas de fallecimiento por consumo de alcohol: cirrosis 
hepática-571; psicosis alcohólicas-291; alcoholismo303; intoxicación alcohólica-E 860. Aunque no todas las muertes por cirrosis están relacionadas con el alcohol, es algo generalmente aceptado que las muertes por cirrosis clasificadas como "cirrosis alcohólicas" $(571,0-571,3)$ no son demasiado fiables, pues sabemos que en muchos países los casos conocidos de esta enfermedad son muy inferiores a los cifras reales. Los análisis de series temporales siguieron el paquete estadístico "STATISTICA".

\section{RESULTADOS}

Índice de mortalidad: las tendencias en los diferentes tipos de índices de mortalidad relacionados con el consumo de alcohol en comparación con las ten- dencias en el nivel de consumo de vodka pueden verse en las figuras 1,2 y 3 . En el período comprendido entre 1970 y 1999, la mortalidad por intoxicación alcohólica aumentó un 4,8 (pasó del 4,9 al 23,4 cada 100.000 habitantes -figura 1). La mortalidad debida a la cirrosis hepática creció un 3,5 (de 3,5 a 12,4 cada 100.000 habitantes -figura 2). La mortalidad debida al alcoholismo o a las psicosis alcohólicas se incrementó diez veces (del 0,3 al 3,4 cada 100.000 habitantes figura 3). Como puede verse, existe un incremento general en el índice de mortalidad relacionada con el consumo de alcohol. Durante la campaña en contra del alcohol realizada entre 1985 y 1988 se observó un importante descenso en el nivel de la mortalidad analizada. Así, el índice de mortalidad por intoxicación alcohólica entre 1984 y 1986 descendió un 46\% (del 13,8 al 7,5 cada 100.000 habitantes). El índice de mor-

Figura 1. Nivel de mortalidad por intoxicación alcohólica (P); nivel de consumo de vodka per cápita (S).

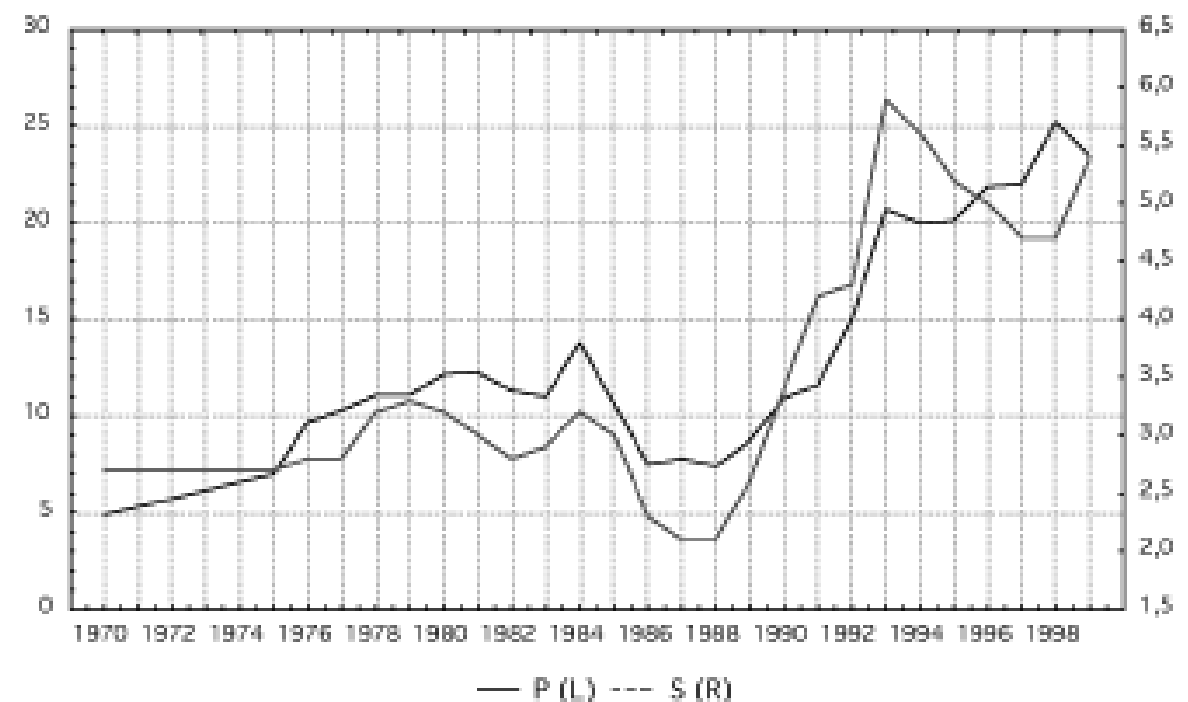

Figura 2. Nivel de mortalidad por cirrosis hepática (C) y nivel de consumo de vodka per cápita (S).

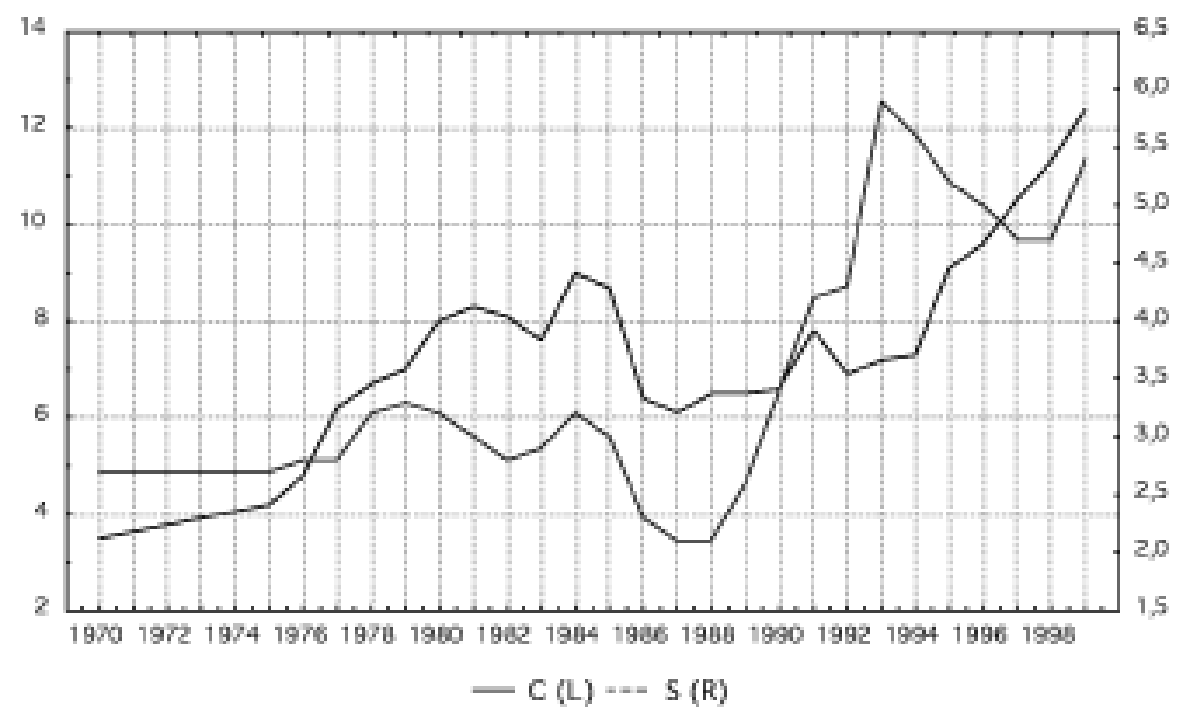


Figura 3. Nivel de mortalidad por alcoholismo y psicosis alcohólicas (AP) y nivel de consumo de vodka per cápita (S).

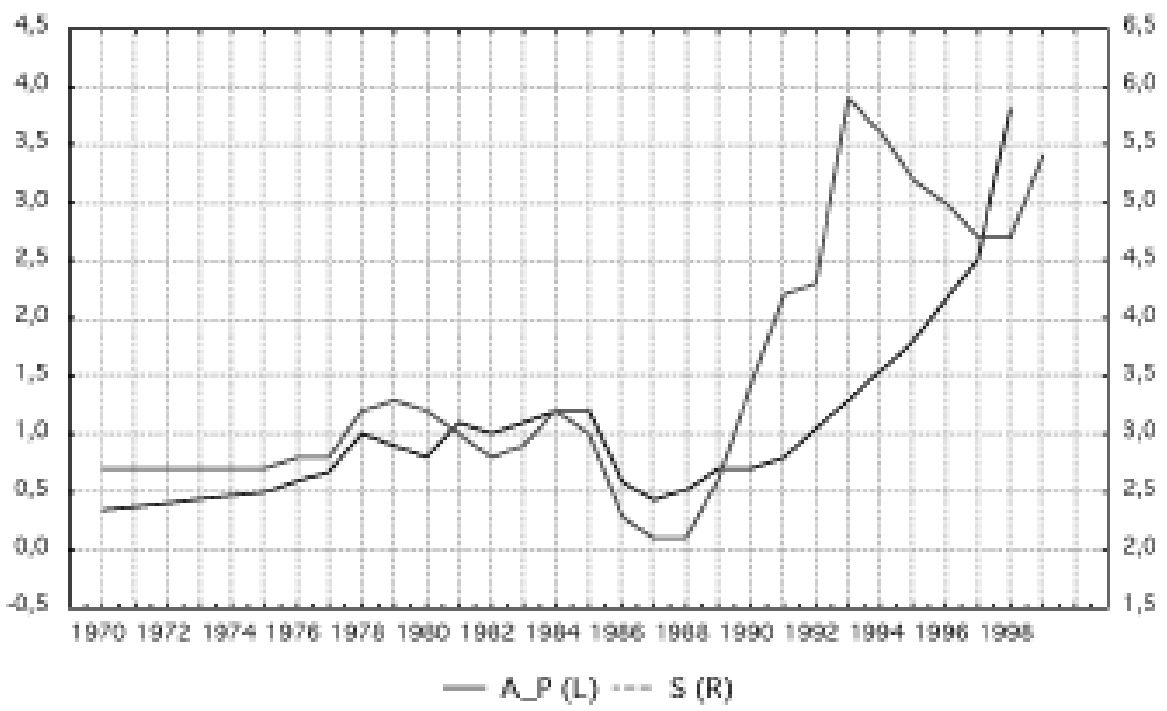

talidad por cirrosis hepática entre 1984 y 1987 descendió un 32\% (del 9 al 6,1 cada 100.000 habitantes). El índice de mortalidad por alcoholismo y psicosis alcohólicas descendió entre 1984 y 1987 un 64\% (del 1,2 al 0,43 cada 100.000 habitantes).

Consumo de alcohol: en la dinámica del nivel de consumo de alcohol per cápita entre 1970 y 1999, podemos distinguir diferentes períodos: 1) de 1970 a 1979; 2) de 1981 a 1983; 3) de 1984 a 1987; 4) de 1987 a 1999 (figura 4). En el primer período, el nivel de consumo aumentó de 6,4 a 10,1 litros, lo que significa un incremento del 58\%. Entre 1981 y 1983, este índice descendió de 10,2 a 9,6 litros. Entre 1985 y 1987, el nivel de consumo de alcohol siguió disminuyendo. En 1987, alcanzó las cotas mínimas de todo el período estudiado. La cifra era de 4,4 litros, lo que significaba un descenso del 55\%. Entre 1988 y 1999, el nivel de consumo de alcohol aumentó un 2,1 (de 4,6 a 9,7 litros). En 1993 se produjo un brutal incremento en el nivel de consumo de alcohol; si lo comparamos con 1992, el índice creció en 1,8 litros, lo que significaba un $30 \%$. Y ese aumento fue debido principalmente al consumo de vodka. El incremento del nivel total de consumo de alcohol en la década de 1970 puede explicarse por el papel predominante del vino, cuyo consumo aumentó 1,9 veces (de 3,1 a 5,9 litros) en el

Figura 4. Tendencias en el consumo de distintos tipos de bebidas alcohólicas per cápita

(T- nivel total de consumo de alcohol; S- nivel de consumo de vodka; W- nivel de consumo de vino; B- nivel de consumo de cerveza).

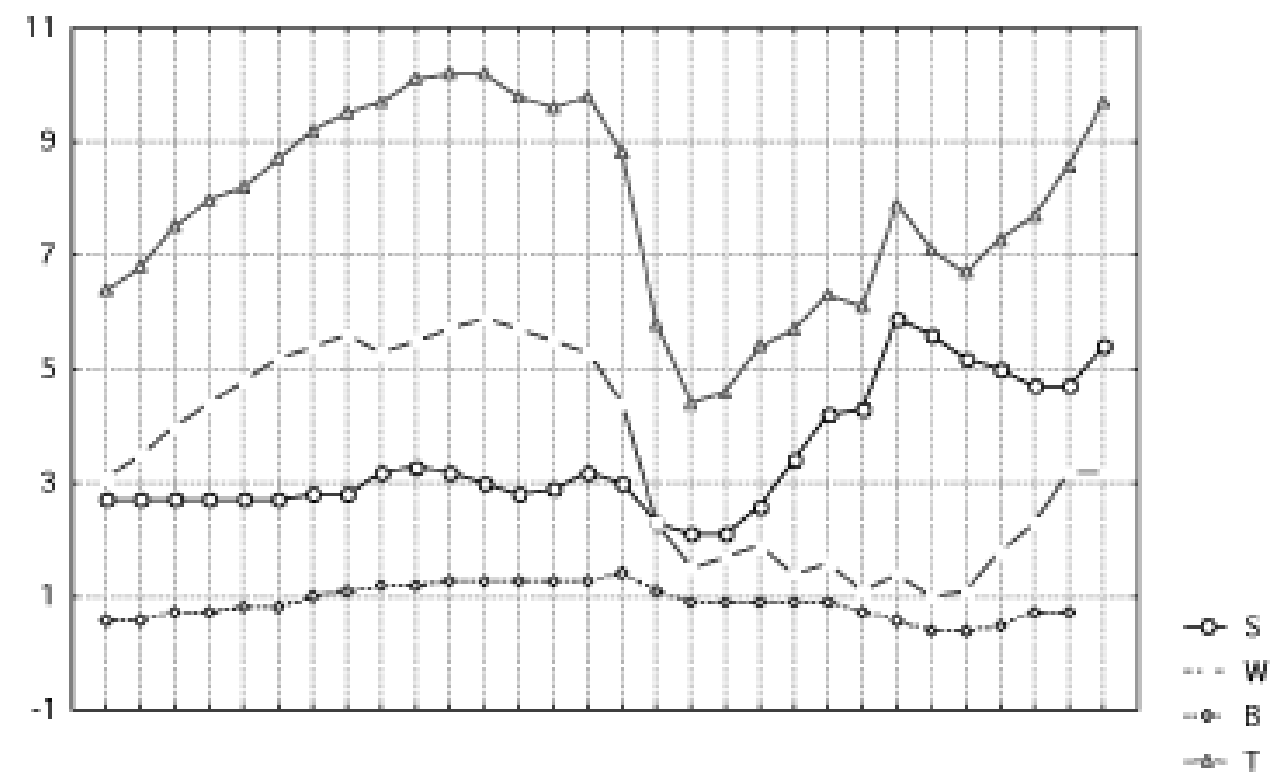


Tabla 1. Resultados del análisis de correlación

\begin{tabular}{|l|c|c|c|c|c|c|c|c|}
\hline & \multicolumn{2}{|c|}{$\mathrm{S}$} & \multicolumn{3}{c|}{ W } & \multicolumn{2}{c|}{$\mathrm{B}$} & \multicolumn{2}{c|}{$T$} \\
\cline { 2 - 8 } & $\mathrm{r}$ & $\mathrm{p}$ & $\mathrm{r}$ & $\mathrm{p}$ & $\mathrm{r}$ & $\mathrm{p}$ & $\mathrm{r}$ & $\mathrm{p}$ \\
\hline $\mathrm{P}$ & $0,90^{*}$ & 0,0001 & $-0,35$ & 0,09 & $-0,04$ & 0,84 & 0,08 & 0,72 \\
$\mathrm{C}$ & $0,71^{*}$ & 0,01 & $-0,11$ & 0,60 & 0,12 & 0,45 & 0,17 & 0,43 \\
$\mathrm{AP}$ & $0,76^{*}$ & 0,001 & 0,14 & 0,52 & $-0,55$ & 0,06 & 0,18 & 0,32 \\
\hline
\end{tabular}

P- mortalidad por intoxicación alcohólica; C- mortalidad por cirrosis hepática; AP- mortalidad por alcoholismo o psicosis alcohólicas; Snivel de consumo de vodka per cápita; W- nivel de consumo de vino per cápita; B- nivel de consumo de cerveza per cápita; T- nivel total de consumo de alcohol.

período comprendido entre 1970 y 1981. Entre 1982 y 1994, el consumo de vino descendió de 5,7 a 1 litro per cápita. El nivel de consumo de vodka per cápita entre 1970 y 1999 se duplicó (de 2,7 a 5,4 litros per cápita). En el período comprendido entre 1980 y 1982, este índice descendió un 12,5\% (de 3,2 a 2,8 litros per cápita). Entre 1984 y 1987, este índice descendió un 34\% (de 3,2 a 2,1 litros per cápita). Entre 1988 y 1999, el nivel de consumo de vodka se incrementó 2,6 veces (de 2,1 a 5,4 litros). El nivel de cerveza consumida en el mismo período era prácticamente estable. Una dinámica opuesta en el consumo de vodka, por una parte, y de vino, por otra, cambió la estructura de consumo del alcohol, en la que pasó a predominar la bebida alcohólica de mayor graduación.

Análisis: el resultado del análisis de correlación aparece en la tabla 1. Como puede verse, la correlación de Pearson indica un grado mayor de covariación entre el nivel de consumo de vodka per cápita y los niveles de mortalidad relacionada con el alcohol: mortalidad por intoxicación alcohólica, cirrosis hepática, alcoholismo y psicosis alcohólicas. Al mismo tiempo, apenas se observa la menor correlación entre el nivel total de consumo de alcohol y el índice de mortalidad relacionada con el alcohol. A través de una función de correlación cruzada se encontró que el índice de intoxicación alcohólica estaba directamente relacionado con el nivel de consumo continuado de vodka $(r=$ $0,90)$. El índice de mortalidad por cirrosis hepática correlaciona con el nivel de consumo continuado de vodka $(r=0,71)$. El índice de mortalidad por alcoholismo y psicosis alcohólicas está directamente asociado al nivel de consumo continuado de vodka $(r=0,76)$.

\section{DISCUSIÓN}

En lo que se refiere al consumo de alcohol, los resultados de la investigación mostraron que éste variaba de un período a otro. Estas variaciones se hallaban en gran medida determinadas por la política estatal respecto al alcohol. Las medidas restrictivas que se tomaron durante las campañas en contra del alcohol de 1981-1983 y de 1985-1988 condujeron a un descenso en el nivel de consumo de alcohol y, por el contrario, la liberalización de la política del alcohol en 1990 se vio acompañada del incremento en el nivel de consumo de vodka (alcohol de alta graduación). Desde el punto de vista de la salud pública, las campañas en contra del alcohol tuvieron un efecto enormemente positivo, que quedó demostrado con el descenso del índice de mortalidad relacionada con el consumo de alcohol. En términos generales, los resultados de la investigación confirman el punto de vista comúnmente aceptado de que la política estatal puede regular el nivel de consumo de alcohol y, por consiguiente, el índice de mortalidad relacionada con el alcohol. En cuanto a los análisis de series temporales, en general, la evidencia descriptiva sugiere una fuerte asociación entre el nivel de consumo de vodka y los diferentes tipos de índice de mortalidad relacionada con el alcohol. De hecho, la asociación casi inmediata entre el consumo de licores y unas consecuencias tanto agudas (intoxicación alcohólica) como crónicas (cirrosis hepática, alcoholismo y psicosis alcohólicas) resulta muy interesante. Merece la pena destacar que los problemas agudos relacionados con el alcohol están mucho más estrechamente vinculados al modelo de consumo de alcohol que al nivel total de consumo. Al mismo tiempo, el nivel de los problemas crónicos relacionados con el alcohol viene determinado por el nivel total de consumo de alcohol. Debemos subrayar que Bielorrusia está dentro del país con el nivel más elevado de consumo de alcohol per cápita (Razvodovsky, 2000). Asimismo, el consumo ocasional de bebidas alcohólicas de alta graduación en grandes cantidades es el modelo predominante de consumo. La combinación del elevado nivel de consumo de alcohol per cápita y el modelo de consumo que persigue la embriaguez ha tenido como consecuencia que, en Bielorrusia, el alcohol repercuta enormemente en el índice de mortalidad relacionada con su consumo. Tal vez sea interesante señalar en este contexto que Bielorrusia tiene un elevado índice de mortalidad por problemas agudos (intoxicación alcohólica), pero un índice relativamente bajo de problemas asociados a largos períodos de consumo de alcohol. Esta eviden- 
cia empírica indica que, en los países donde la cultura del alcohol se decanta por el consumo de vodka, existe una elevada mortalidad por intoxicación alcohólica. Estos países, asimismo, suelen tener un índice relativamente bajo de mortalidad por cirrosis hepática (Harkin y col., 1995).

Así, pues, los resultados del presente estudio confirman la idea de que los índices de mortalidad relacionados con el consumo de alcohol tienden a ser más sensibles a los cambios en el consumo de vodka per cápita que al nivel total de consumo de alcohol. La principal evidencia para llegar a esta conclusión es la asociación directa y significativa entre los índices de mortalidad relacionados con el consumo de alcohol y el nivel de consumo de vodka per cápita. El aumento en el nivel de consumo de las bebidas alcohólicas de alta graduación conduce al incremento del índice de mortalidad por problemas tanto crónicos como agudos. El resultado de este estudio indica también que la mortalidad por intoxicación alcohólica es un indicador preferente de los daños relacionados con el alcohol en los países donde predomina el modelo de consumo que persigue la embriaguez. Este artículo se suma a la cada vez mayor evidencia científica de que un porcentaje sustancial de la mortalidad relacionada con el consumo de alcohol en Bielorrusia es debido a los problemas agudos que se derivan del hecho de consumir alcohol para emborracharse.

\section{BIBLIOGRAFÍA}

Edwards, G., (1994) Alcohol Policy and Public Good. Oxford. Harkin, A.M., Anderson, P., Lehto, J. (eds.). (1995) Alcohol in Europe- a health perspective. Copenhagen.

Kerr, W.C., Fillimore, K.M., Marrvy, P. (2000) Beveragespecific alcohol consumption and cirrhosis mortality in a group of English-speaking beer-drinking countries. Addiction. 2000; 95(3):339-46.

Lelbach, W. K. (1976) Epidemiology of alcoholic liver disease. In: Popper, H. and Schaffer, F. (eds.) Progress in Liver Disease. New York. Grune and Stratton. Vol. 5:494-515.

Makela, K., Room, R., Single, E., Sulkunen, P., Walsh, B. (1981) Alcohol, Society and the State. Toronto. Addiction Research Foundation.

Norstrom, T. (1987) The abolition of the Swedish alcohol rationing system: Effects on consumption distribution and cirrhosis mortality. British Journal of Addiction. 82: 633-642.

Poikolainen, K. (1977) Alcohol poisoning mortality in four Nordic countries. Helsinki. Finnish Foundation for Alcohol Studies.

Ramstedt, M. (2001) Comparative studies on alcohol-related problems in postwar Western Europe. Stockholm. 9-10.

Ramstedt, M. (2001) Per capita alcohol consumption and liver cirrhosis mortality in 14 European countries. Addiction. 96(1): 19-34.

Razvodovsky, Y.E. (2000) Alcohol-related problems in Belarus. Alcologia. 12: 10-14.

Skog, O.J. (1984) The risk function for liver cirrhosis from lifetime alcohol consumption. Journal of studies on Alcohol. 45:199-208.

Skog, O.J. (1988) Alcohol related problems in Norway: Patterns and trends. In: Alcohol and Drugs: The Norwegian Experience. Skog, O.J., Waahlberg, R. (eds.) Norway. p. 47-69.

Smart, R.G., Mann, R.E. (1992) Alcohol and the epidemiology of liver cirrhosis. Alcohol Health Res. World 16: 217-222.

Thorsen, T. (1990) Hundrede ars alcoholmisbrug. Alcohol-og Nakotikaradet. Copenhagen.

Vaillant, G. E. (1983) The Natural History of Alcoholism: Causes, Patterns and Paths to Recovery. Harvard University Press. 